\title{
Low-temperature plasma radiofrequency ablation for the management of refractory cluster headache
}

\author{
Yuna Guo, Xiaoping Wang, Jingjing Bian, Zhi Dou, Liqiang Yang, Jiaxiang Ni, Yuanzhang Tang \\ Pain Department, XuanWu Hospital, Capital Medical University, Beijing, China
}

Videosurgery Miniinv 2021; 16 (2): 362-368

DOI: https://doi.org/10.5114/wiitm.2020.100739

\begin{abstract}
Introduction: Although the sphenopalatine ganglion (SPG) has been considered a site of therapeutic potential for cluster headache (CH), the optimal technique of SPG is still to be determined. Low-temperature plasma radiofrequency ablation (LTPRA) has been proposed as an alternative treatment for several neuropathic pain diseases.

Aim: To evaluate the effect of LTPRA of SPG in treating chronic and episodic $\mathrm{CH}$.

Material and methods: The patients with $\mathrm{CH}$, who achieved temporary pain relief following SPG block, treated using LTPRA between January 2015 and October 2017 were reviewed. Seventy-six patients were included: 50 patients suffered from episodic $\mathrm{CH}$ and the remaining 26 patients from chronic $\mathrm{CH}$. The primary outcomes were clinical improvement rate, defined as the percentage of partial and complete pain relief results at 1 day, 12 months, and 24 months of follow-up after the operation.

Results: Clinical improvement rates were $92.3 \%, 92.3 \%$ and $73.1 \%$ in chronic $\mathrm{CH}$ and $73.1 \%, 84 \%$ and $68 \%$ in episodic $\mathrm{CH}$ at each follow-up time point, respectively. 3 chronic $\mathrm{CH}$ patients and 7 episodic $\mathrm{CH}$ patients showed no pain relief after the operation. Drooping eyelids were found in 2 cases, one recovered at the 3-month follow-up but another one did not in the 24-month follow-up. No serious complications occurred intraoperatively or postoperatively. Conclusions: LTPRA can be considered an effective and alternative surgical modality in treating patients with chronic and episodic $\mathrm{CH}$, based on SPG block.
\end{abstract}

Key words: cluster headache, low-temperature plasma radiofrequency ablation, numeric rating scale, pain, sphenopalatine ganglion.

\section{Introduction}

Cluster headache $(\mathrm{CH})$ is a kind of extremely severe unilateral headache attacks, accompanied by ipsilateral autonomic symptoms [1]. There are two types of $\mathrm{CH}$, episodic and chronic. Episodic $\mathrm{CH}$ attacks have a duration of $15-180 \mathrm{~min}$, last for several weeks to 1 year and are followed by remission periods of at least 3 months. Chronic $\mathrm{CH}$ recurrent attacks occur for longer than 1 year with no pain-free periods or with remission periods less than 1 month. Medication is the first choice for patients with $\mathrm{CH}$.
For patients who have persistent pain despite drug therapy, or who are unable to tolerate the side effects of drugs, several alternative surgical modalities of sphenopalatine ganglion (SPG) for $\mathrm{CH}$ have emerged, including block [2, 3], radiofrequency [4-6] and electrical stimulation $[7,8]$ procedures. Despite SPG block obtaining a high efficacy rate, symptomatic relief was not permanent, and further radiofrequency thermocoagulation (RFT) of SPG to obtain permanent pain relief has been suggested [9]. However, RFT was used to treat $\mathrm{CH}$ with unsatisfactory results [10].

\section{Address for correspondence}

Yuanzhang Tang PhD, MD, Pain Department, XuanWu Hospital, Capital Medical University, Beijing, China,

e-mail: medtangyz@hotmail.com 
We have reported that low-temperature plasma radiofrequency ablation (LTPRA) obtained better clinical outcomes than RFT in treatment of thoracic neuropathic pain [11], phantom limb pain [12] and trigeminal neuralgia [13]. The mechanisms of LTPRA are based on molecular dissociation rather than heat damage as in RFT $[10,14]$. Nevertheless, the efficacy and safety of SPG LTPRA for $\mathrm{CH}$ are still unclear.

\section{Aim}

This study retrospectively evaluated the efficacy and safety of patients with $\mathrm{CH}$ treated using LTPRA in the pain department of our hospital between January 2016 and October 2017. Special focus was placed on the value of SPG block to choose appropriate $\mathrm{CH}$ patients to receive SPG LTPRA and to help physicians apply better treatment modalities.

\section{Material and methods}

We reviewed the patients with $\mathrm{CH}$ treated with LTPRA. The study was approved by the Ethics Committee of our hospital and prior written informed consent to LTPRA was obtained in all patients.

A total of 76 patients were diagnosed with $\mathrm{CH}$ (50 patients with episodic $\mathrm{CH}$, the remaining 26 patients with chronic $\mathrm{CH}$ ) and received LTPRA. All patients had received conservative medication treatments such as rizatriptan, carbamazepine or other available analgesics; when these drugs were ineffective and the numeric rating scale (NRS) score used to assess the pain degree was still higher than 6 , it was considered intractable and they received SPG block during pain attacks. If the SPG block test was positive, which indicates that the NRS score decreases more than $50 \%$ in $30 \mathrm{~min}$, the patient was suggested to receive LTPRA. If the SPG block was negative, the patient was suggested to look for other treatment methods. Each episodic $\mathrm{CH}$ patient was treated within 7 days after the onset of the first symptom of the cluster period.

Those patients with tumors, infection, coagulation disorders, pregnancy, lactation, severe organ disease, and mental disorders were excluded.

\section{SPG block}

The patient was placed in the supine position. Each patient's vital signs were monitored. The bilateral pterygopalatine fossa overlaps under standard lateral fluoroscopy vision. After 1\% lidocaine local anesthesia, a needle (22-gauge, $15 \mathrm{~cm}$ ) was inserted under the zygomatic arch and advanced superiorly and medially to the middle of the pterygopalatine fossa under fluoroscopy guidance (Photo $1 \mathrm{~A}$ ). Then under anteroposterior view, the needle tip was lateral to the middle turbinate (Photo $1 \mathrm{~B}$ ). We injected $1 \mathrm{ml}$ of $1 \%$ lidocaine slowly and recorded the NRS.

\section{SPG LTPRA procedure}

The LTPRA procedure was performed another day after the block and under computed tomography (CT) guidance. Patients were placed in supine position and vital signs were monitored. In brief, the puncture channel to the pterygopalatine fossa was planned on the CT scan. An 18-gauge, 15-cm Tuohy needle was inserted through the designed puncture channel to the pterygopalatine fossa (Photo $1 \mathrm{C}$ ). The correct position of the needle was verified by CT scan. After inserting the cryogenic plasma ablation needle, sensory stimulation was started as we reported before [13] (Photo $1 \mathrm{D})$. The positive electrode of the nerve stimulator was connected with the anode of the tail end of the cryogenic plasma ablation needle and the negative electrode was connected to the patient's skin. The stimulus intensity was gradually increased by $2 \mathrm{~Hz}$ and $0.1 \mathrm{~ms}$. If the position is correct, the paresthesia will be perceived at the endonasal level under $0.5 \mathrm{~V}$. After that, intravenous anesthesia with propofol was administered, and the cryogenic plasma ablation needle was connected to the low-temperature plasma multi-function operating system (SM-D380C, Xi'an Surgical Medical Technology Co., Ltd., China). Low-temperature plasma radiofrequency ablation was then performed in mode 2 (45 W) for 30 s twice, with a 30 s interval.

\section{Data collection and follow-up}

Follow-up was performed via telephone interviews. The medical records of all patients were reviewed and follow-up data were gathered on the demographics, including age, sex, pain location (right or left), duration of headache, duration of cluster periods (months), number of clusters per year, attack duration (hours), pain intensity during attack before surgery, number of attacks per day and remission period duration, side affected, pain intensity at follow-up day. Adverse effects and 

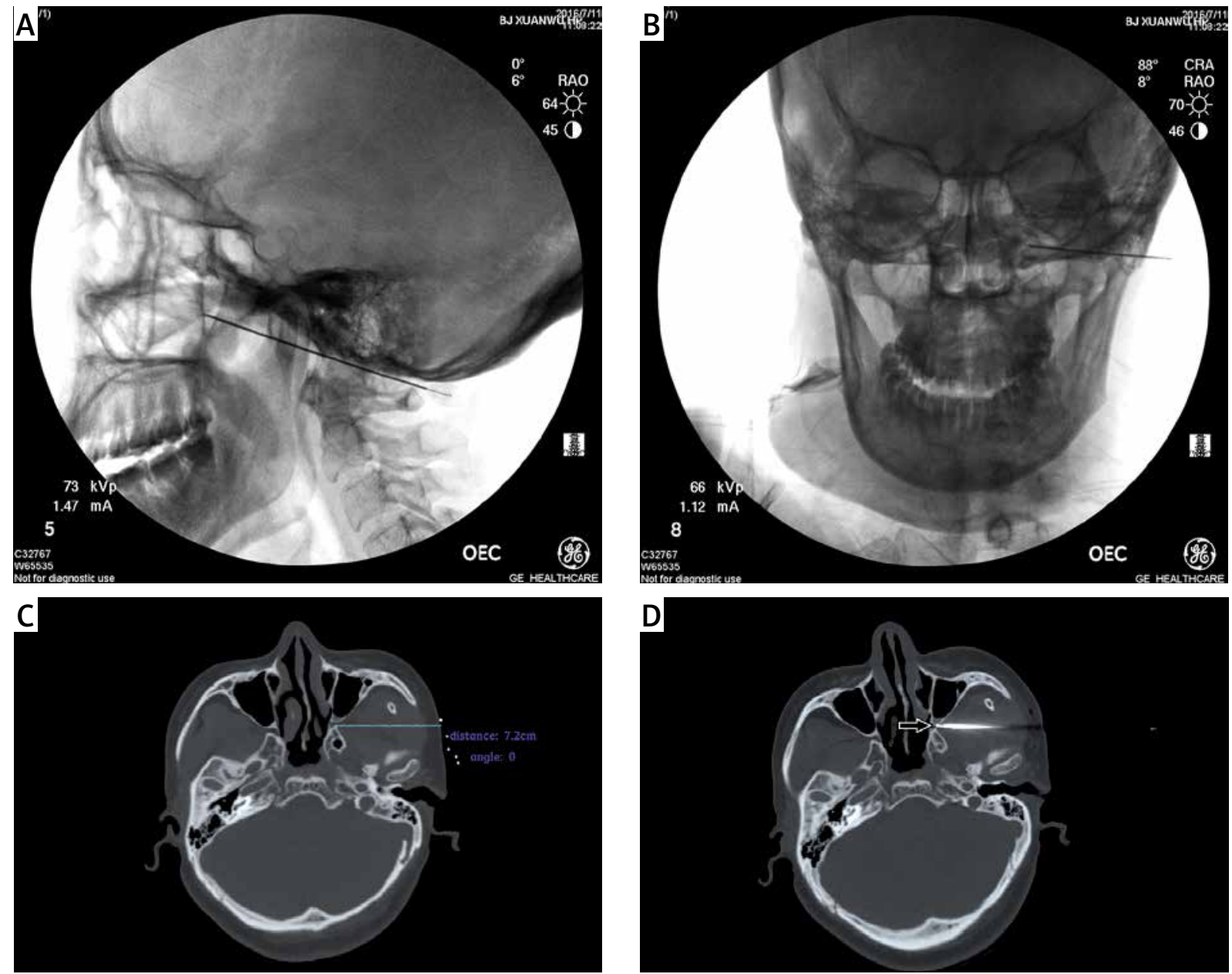

Photo 1. The correct placement of the tip of the block (A, B) or Tuohy (C, D) needle in the pterygopalatine fossa. Lateral view (A) and anteroposterior view (B) guided by fluoroscopy. (C) and (D) guided by CT scans. The black arrow indicates the tip of the cryogenic plasma ablation needle

complications related to the procedure were also recorded.

The primary outcomes were clinical improvement rate, defined as the percentage of partial and complete pain relief results at 1 day, 12 months and 24 months after the operation. NRS was used to measure pain intensity. To perform statistical analysis of pain intensity, the results were grouped as follows: complete pain relief was indicated if the patient stopped experiencing pain and parasympathetic symptoms and the pharmacologic treatment was then terminated; partial pain relief was defined as a decrease in the number of attacks or the intensity of the attacks resulting in a reduced pharmacologic need; no pain relief was indicated if the post-operative pain condition was at the same level as the pre-operative condition [3].

\section{Statistical analysis}

The statistical analysis was carried out using SPSS 22.0 (SPSS Inc. United States). The measurement data were presented as mean \pm standard deviation. The NRS score, cluster period and remission period of patients before and after the treatment were compared using Student's paired $t$-test. A $p$-value $<0.05$ indicated statistical significance.

\section{Results}

In the study, after testing positive for SPG block, 76 patients suffering from $\mathrm{CH}$ underwent a CT-guided LTPRA operation. The basic characteristics of the patients are shown in Table I. The most common parasympathetic symptoms of $\mathrm{CH}$ patients are lacrimation and conjunctival injection. 


\section{Treatment effects}

Clinical improvement was observed in 24 (92.3\%) chronic $\mathrm{CH}$ patients and 48 (96\%) episodic $\mathrm{CH}$ patients, with no improvement in 4 patients at 1 day after the operation. These 4 patients with recalcitrant symptoms refused an additional operation.

In patients with chronic $\mathrm{CH}$, the clinical improvement rate was $92.3 \%$ and $73.1 \%$ at 12 and 24 months after the operation, with 3 patients having pain relapse at $13,15,16$ months, respectively. These 3 patients, who had pain recurrence after the first operation, received the second LTPRA. Then 2 patients had partial pain relief and 1 patient had no pain relief during the follow-up. Particularly, 6 patients achieved complete pain relief, experienced pain and parasympathetic symptoms stopped and the drug was terminated after the first operation at 24 months (Table II).

In patients with episodic $\mathrm{CH}$, the clinical improvement rate was $84 \%$ and $68 \%$ at 12 and 24 months after the operation, with 6 patients and 8 patients having pain relapse within 12 and 24 months, respectively. Of these 14 patients obtained clinical improvement after the first operation, 11 patients received the second LTPRA and 3 patients sought additional treatment in a different hospital. Of these 11 patients after the second operation, 9 patients had partial pain relief and 2 patients had no pain relief during the follow-up. Particularly, 18 patients had complete pain relief, experienced pain and parasympathetic symptoms stopped and the drug was terminated after the first operation at 24 months (Table III).

In all, 3 chronic $\mathrm{CH}$ patients and 7 episodic $\mathrm{CH}$ patients were not responsive to the SPG LTPRA during follow-up, who were treated with increasing doses of analgesic drugs and looked for treatment in a different hospital.

The NRS decreased from 7.25 (pre-operation) to $2.5,3.63$ and 3.23 in chronic $\mathrm{CH}$ patients and decreased from 7.13 (pre-operation) to 3.26, 4.36 and 4.84 in episodic $\mathrm{CH}$ patients at 1 day, 12 months and 24 months after LTPRA, respectively. The mean value of pain intensity shows a significant reduction at each follow-up time point compared to pre-operation $(p<0.05)$ in these two groups of patients, which indicates that LTPRA is an effective treatment for $\mathrm{CH}$. All the episodic $\mathrm{CH}$ patients with improvement in results have presented a significant decrease of

Table I. Clinical characteristics of $\mathrm{CH}$ patients $(n=76)$

\begin{tabular}{|lcc|}
\hline Characteristics & $\begin{array}{c}\text { Episodic } \\
\text { CH }\end{array}$ & $\begin{array}{c}\text { Chronic } \\
\text { CH }\end{array}$ \\
\hline Patient no. & 50 & 26 \\
\hline Gender (male/female) & $22 / 28$ & $15 / 11$ \\
\hline Age [years] & $48.4 \pm 13.3$ & $48.5 \pm 18.7$ \\
\hline location side (left/right) & $24 / 26$ & $11 / 15$ \\
\hline Duration of headache [years] & $11.7 \pm 9.3$ & $6.8 \pm 9.9$ \\
\hline Duration of cluster periods [months] & $3.4 \pm 1.7$ & - \\
\hline No. of clusters [per year] & $1.8 \pm 0.6$ & - \\
\hline Attack duration [h] & $2.3 \pm 0.7$ & $2.1 \pm 1.2$ \\
\hline No. of attacks [per day] & $3.2 \pm 1.1$ & $2.2 \pm 1.6$ \\
\hline \begin{tabular}{l} 
Pre-operation NRS \\
\hline $\begin{array}{l}\text { Parasympathetic symptoms } \\
\text { (no. of patients) \%: }\end{array}$
\end{tabular} & $(3.13 \pm 0.65$ & $7.25 \pm 0.60$ \\
\hline \begin{tabular}{l} 
Lacrimation \\
\hline Moniosis
\end{tabular} & $(33) 66 \%$ & $(19) 73.1 \%$ \\
\hline
\end{tabular}

- not applicable, $\mathrm{CH}$-cluster headache, NRS - numeric rating scale.

Table II. Pain relief rate of patients with chronic $\mathrm{CH}$ after LTPRA at each follow-up time point

\begin{tabular}{|lccc|}
\hline Follow-up time point & 1 day & 12 months & 24 months \\
\hline Complete pain relief \% (no. of patients) & $42.3 \%(11)$ & $30.8 \%(8)$ & $23.1 \%(6)$ \\
\hline Partial pain relief \% (no. of patients) & $50 \%(13)$ & $61.5 \%(16)$ & $57.7 \%(15)$ \\
\hline No pain relief \% (no. of patients) & $7.7 \%(2)$ & $7.7 \%(2)$ & $19.2 \%(5)$ \\
\hline
\end{tabular}

$\mathrm{CH}$ - cluster headache, LTPRA - low-temperature plasma radiofrequency ablation. 
Table III. Pain relief rate of patients with episodic $\mathrm{CH}$ after LTPRA at each follow-up time point

\begin{tabular}{|lccc|}
\hline Follow-up time point & 1 day & 12 months & 24 months \\
\hline Complete pain relief \% (no. of patients) & $48 \%(24)$ & $44 \%(22)$ & $36 \%(18)$ \\
\hline Partial pain relief \% (no. of patients) & $48 \%(24)$ & $40 \%(20)$ & $32 \%(16)$ \\
\hline No pain relief \% (no. of patients) & $4 \%(2)$ & $16 \%(8)$ & $32 \%(16)$ \\
\hline
\end{tabular}

$\mathrm{CH}$ - cluster headache, LTPRA - low-temperature plasma radiofrequency ablation.

post-operative cluster and remission duration at 12 and 24 months compared to pre-operation $(p<0.05$, Table IV).

\section{Complications}

Palate or teeth numbness after the LTPRA operation occurred in 20 (40\%) patients with episodic $\mathrm{CH}$ and 10 (38.5\%) patients with chronic $\mathrm{CH}$. Fortunately, the intensity in all these patients was mild to moderate and all cases lasted less than 12 months with complete resolution. Ptosis was found in 2 cases with episodic $\mathrm{CH}, 1$ recovered in 3-month but another 1 did not during 24-month follow-up. No serious complications occurred intraoperatively or postoperatively.

\section{Discussion}

Our results showed that LTPRA is an effective and safe treatment for chronic and episode $\mathrm{CH}$ patients. Presence of ipsilateral autonomic symptoms is a key factor to diagnose $\mathrm{CH}$. We first analyzed the presence of autonomic symptoms and found that lacrimation and conjunctival injection are the most common symptoms. This analysis is beneficial for $\mathrm{CH}$ diagnosis in clinical practice. SPG is one of the potential therapeutic targets for $\mathrm{CH}$. We demonstrated that SPG block performed before LTPRA surgery to choose adaptive SPG-dominated $\mathrm{CH}$ patients is an effective method to achieve better results. Additionally, the variation in duration of cluster and remission of episodic $\mathrm{CH}$ patients also means that there is excellent pain control after the procedure. Episodic cluster headaches occur over periods from
7 days to 1 year separated by pain-free periods lasting at least 1 month. In episodic $\mathrm{CH}$ patients, the average cluster period before the operation was 3.4 \pm 1.7 months and the duration between the onset of symptoms and the operation was less than 7 days, indicating that the cluster period could not end before the treatment.

Salgado-Lopez et al. [10] observed 13 patients with $\mathrm{CH}$ and reported that only $8(61.5 \%)$ patients obtained clinical improvement after SPG RFT. Interestingly, Narouze et al. [15] conducted a study to examine the treatment effects of SPG RFT in $15 \mathrm{CH}$ patients, who had positive responses to SPG block before RFT. They noted remarkable improvement in the pain disability index, attack intensity and frequency at a mean follow-up period of 18 months. Our study agrees with Narouze's report; an SPG block should be performed for patients with $\mathrm{CH}$ before the operation to obtain better clinical outcomes. Maybe this is the reason that the results of Narouze and our study are better than those of Salgado-Lopez $\mathrm{L}$.

The pathophysiology of $\mathrm{CH}$ is not fully elucidated [16]. The traditional mechanism [17] of cluster headache arises from the reflex activation of the trigeminal-autonomic reflex pathway through parasympathetic outflow through the sphenopalatine ganglion (SPG), resulting in vasodilatation and parasympathetic activation. Costa et al. [2] conducted a double-blind placebo-controlled study to compare the SPG block effects of cocaine and lidocaine for nitroglycerin-induced cluster headache which found that most patients responded to pain relief. In recent years, there has emerged evidence for the involvement of the hypothalamus in $\mathrm{CH}$, and this evidence

Table IV. Variation in cluster and remission periods of episodic CH patients' response to LTPRA at follow-up.

\begin{tabular}{|lccc|}
\hline Follow-up time point & Pre-operation & $\begin{array}{c}\text { Post-operation } \\
(12 \text { months })\end{array}$ & $\begin{array}{c}\text { Post-operation } \\
(24 \text { months })\end{array}$ \\
\hline Duration of clusters [months] & $3.4 \pm 1.7$ & $0.4 \pm 0.6^{*}$ & $0.9 \pm 0.7^{\star}$ \\
\hline Duration of remission [months] & $7.2 \pm 2.4$ & $11.8 \pm 1.9^{*}$ & $10.7 \pm 2.1^{\star}$ \\
\hline
\end{tabular}

$\mathrm{CH}$ - cluster headache, LTPRA - low-temperature plasma radiofrequency ablation. ${ }^{\star} P<0.05$ vs. pre-operation. 
was supported by the therapeutic effect of targeting the hypothalamus gray through deep brain stimulation $[7,8]$. So, SPG and the hypothalamus may be two potential therapeutic targets for $\mathrm{CH}$. SPG block is an effective and safe way to evaluate the clinical symptoms related to SPG and can be used to ensure effectiveness of SPG surgery. In our study, 10 patients were not responsive to the SPG LTPRA during follow-up; their symptoms may be partly related to hypothalamus. Further deep brain stimulation may benefit them.

LTPRA is an innovative method for the treatment of neuralgia, which is a different RFT because it is responsible for dissociation of intercellular bonds in tissues, not coagulation at high temperature [18]. In trigeminal neuralgia treatment [13], LTPRA has obtained a similar pain relief rate; however, facial numbness is much less common than RFT. A similar result was also reported for thoracic nerve pain [11]; it was found that $80 \%$ of patients achieved obvious pain relief with slight numbness. Permanent hypoesthesia or numbness of the palate, cheek, and teeth was also reported after SPG RFT for treating $\mathrm{CH}$ [15, 19]. However, no permanent numbness occurred in our study; 30 patients had temporary mild numbness of the teeth or palate and in all it disappeared gradually in 12 months, which indicated that this side effect could be avoided or mitigated through the LTPRA procedure. Unfortunately, 1 patient had ptosis after a 24-month follow-up, which may be related to sympathetic disruption. A multi-center clinical study is strongly recommended in the future to perform a randomized controlled study and better evaluate the effectiveness and safety of LTPRA and RFT for $\mathrm{CH}$.

Our department reported the effectiveness and safety of LTPRA in the treatment of refractory cluster headache a few months ago [20]. However, we made some innovations which are unique and valuable in this report. Firstly, we fully described parasympathetic symptoms in chronic and episodic patients. $\mathrm{CH}$ is a rare severe headache accompanied by parasympathetic symptoms. These results may be beneficial for diagnosis of $\mathrm{CH}$ in the clinic. Secondly, we thoroughly reported the effectiveness and safety of both chronic $\mathrm{CH}$ and episodic $\mathrm{CH}$, which may be valuable for doctors in treating different types of patients. Thirdly, we chose appropriate patients with $\mathrm{CH}$ for LTPRA technique based on SPG block. SPG block was used to choose appropriate $\mathrm{CH}$ patients to receive SPG LTPRA and to help physicians make wise treatment modali- ty choices. Patients negative for SPG block may have other pathophysiological mechanisms. Interestingly, our results are similar to a previous study, which did not use SPG block before surgery. This phenomenon would be addressed in a further control study (with or without SPG block on effectiveness of LTPRA).

The present study has several limitations. It was a single-center retrospective study, and it lacks a control group. The results derived from this 24-months follow-up study may not be generalizable to different patient populations. A longer-term follow-up should be performed to further evaluate its benefits and complications.

\section{Conclusions}

In short, we concluded from the current study that LTPRA had satisfactory effectiveness and safety in the treatment of chronic and episodic $\mathrm{CH}$. SPG block, which is the best way to choose adaptive SPG-dominated $\mathrm{CH}$ patients, should be performed before SPG LTPRA.

\section{Acknowledgments}

This study was supported by "Beijing Municipal Administration of Hospitals Incubating Programme" code: PX2020033.

Yuna Guo and Xiaoping Wang contributed to the work equally and should be regarded as co-first authors.

\section{Conflict of interest}

The authors declare no conflict of interest.

\section{References}

1. Headache Classification Committee of the International Headache Society (IHS) The International Classification of Headache Disorders, 3rd edition. Cephalalgia 2018; 38: 1-211.

2. Costa A, Pucci E, Antonaci F, et al. The effect of intranasal cocaine and lidocaine on nitroglycerin-induced attacks in cluster headache. Cephalalgia 2000; 20: 85-91.

3. Sanders M, Zuurmond WW. Efficacy of sphenopalatine ganglion blockade in 66 patients suffering from cluster headache: a 12- to 70-month follow-up evaluation. J Neurosurg 1997; 87: 876-80.

4. Shah RJ, Padalia D. Sphenopalatine Ganglion Radiofrequency Thermocoagulation. StatPearls. Treasure Island (FL) 2019.

5. Loomba V, Upadhyay A, Kaveeshvar H. Radiofrequency ablation of the sphenopalatine ganglion using cone beam computed tomography for intractable cluster headache. Pain Physician 2016; 19: E1093-6. 
6. Fang L, Jingjing L, Ying S, et al. Computerized tomography-guided sphenopalatine ganglion pulsed radiofrequency treatment in 16 patients with refractory cluster headaches: twelve- to 30-month follow-up evaluations. Cephalalgia 2016; 36: 106-12.

7. Leone M, Franzini A, Cecchini AP, et al. Hypothalamic deep brain stimulation in the treatment of chronic cluster headache. Ther Adv Neurol Disord 2010; 3: 187-95.

8. Schoenen J, Di Clemente L, Vandenheede M, et al. Hypothalamic stimulation in chronic cluster headache: a pilot study of efficacy and mode of action. Brain 2005; 128: 940-7.

9. Salar G, Ori C, Iob I, Fiore D. Percutaneous thermocoagulation for sphenopalatine ganglion neuralgia. Acta Neurochir 1987; 84: 24-8.

10. Salgado-Lopez L, de Quintana-Schmidt C, Belvis Nieto R, et al. Efficacy of sphenopalatine ganglion radiofrequency in refractory chronic cluster headache. World Neurosurg 2019; 122: e262-69.

11. Yang LQ, Gong WY, Wang XP, et al. Computed tomography-guided percutaneously controlled ablation of the thoracic paravertebral nerve due to thoracic neuropathic pain. Pain Pract 2017; 17: 792-9.

12. Zeng Y, Wang X, Guo Y, et al. Coblation of femoral and sciatic nerve for stump pain and phantom limb pain: a case report. Pain Practice 2016; 16: E35-41.

13. Li Y, Guo Y, Yang L, Ni J. Comparison of the short-term outcomes after low-temperature plasma radiofrequency ablation (coblation) in the Gasserian ganglion for the treatment of idiopathic trigeminal neuralgia. J Pain Res 2019; 12: 1235-42.

14. Matthews B, Wilkinson M, McEwen P, et al. In vivo arthroscopic temperatures: a comparison between 2 types of radiofrequency ablation systems in arthroscopic anterior cruciate ligament reconstruction-a randomized controlled trial. Arthroscopy 2017; 33: 165-72.

15. Narouze S, Kapural L, Casanova J, Mekhail N. Sphenopalatine ganglion radiofrequency ablation for the management of chronic cluster headache. Headache 2009; 49: 571-7.

16. Wei DY, Yuan Ong JJ, Goadsby PJ. Cluster headache: epidemiology, pathophysiology, clinical features, and diagnosis. Ann Indian Acad Neurol 2018; 21 (Suppl 1): S3-8.

17. Knight YE, Classey JD, Lasalandra MP, et al. Patterns of fos expression in the rostral medulla and caudal pons evoked by noxious craniovascular stimulation and periaqueductal gray stimulation in the cat. Brain Res 2005; 1045: 1-11.

18. Mittal B, Thomas DG. Controlled thermocoagulation in trigeminal neuralgia. J Neurol Neurosurg Psychiatry 1986; 49: 932-6.

19. Narouze SN. Role of sphenopalatine ganglion neuroablation in the management of cluster headache. Curr Pain Headache Rep 2010; 14: 160-3.

20. Wang N, Dou Z, He L, et al. Sphenopalatine ganglion-targeted low-temperature plasma radiofrequency ablation in the treatment of refractory cluster headache. Videosurgery Miniinv 2020; 15: 313-8.

Received: 9.06.2020, accepted: 16.09.2020. 